\title{
Las desigualdades interregionales en México, 1970-1980*
}

\section{María Delfina Ramírez}

En el contexto de los estudios existentes sobre las disparidades regionales en México, en este artículo se realiza un análisis pormenorizado del desarrollo de las desigualdades interregionales entre 1970-1980. Se trata de determinar si ha ocurrido una reducción de las desigualdades entre las regiones mexicanas o si éstas han seguido creciendo como se observó en el periodo 1900-1970. Según las dos regionalizaciones utilizadas, se concluye que en 1970-1980 persiste la tendencia hacia mayores desigualdades regionales en México.

\section{Introduccion}

Algunos estudios empíricos realizados hasta 1970 sobre las desigualdades interregionales en México concluyen que: primero, de 1900 a 1970 ha habido una mejora en las condiciones de vida de los habitantes del país en cada una de las regiones y entidades federativas, pero esa mejora se dio a ritmos desiguales; segundo, la desigualdad económica y social entre las regiones del país en el mismo periodo ha seguido creciendo hasta alcanzar niveles de disparidad interregional sumamente preocupantes. ${ }^{1}$ Para 1970-1980 se ha dicho que parecen apreciarse indicios de una cierta convergencia entre los niveles económicos de las regiones del país (Hernández Laos, 1984).

Parecen existir suficientes elementos en el modelo de crecimiento económico del país, así como en el apoyo del Estado a ese modelo ${ }^{2}$ que sugieren, si no un aumento, al menos la contuinidad del desequilibrio económico y social entre sus sectores de actividad, así como entre sus regiones. Nos parece por tanto de gran importancia desarrollar el estudio de la década 1970-1980, para agregar elementos de juicio respecto al posible inicio de cierta convergencia entre las regiones del país en ese periodo.

En las siguientes páginas realizaremos un análisis pormenorizado de las tendencias de las desigualdades interregionales entre 1970 y 1980 , con

* Este artículo es una versión reducida del estudio sobre las desigualdades interregionales en México, presentado como tesis para optar al grado de Maestro en Desarrollo Urbano, en El Colegio de México, en junio de 1986.

1 Para un análisis comprehensivo al respecto, véase Wilkie (1967), Lemoine (1973), y Unikel, Ruiz y Garza (1976), entre otros.

${ }_{2}^{2}$ La concentración de poder político administrativo en el área urbana más importante del país, con la consecuente concentración de gasto de capital público y privado en la misma, se acentuó notablemente cuando se optó por la expansión industrial, que se tradujo en el crecimiento de actividades urbanas (industria y servicios) en detrimento de los recursos destinados a extensas zonas agrícolas. 
objeto de verificar hasta qué grado ha ocurrido una reducción de las disparidades regionales en México en esta etapa.

\section{Distribución de la actividad económica por entidad federativa}

Tanto en los trabajos mencionados como en la cuantificación propia que presentamos enseguida, el objeto de estudio lo han constituido lo que se puede llamar las "regiones evidentes", que han sido conformadas con base en una demarcación político-administrativa: las entidades federativas del país, o bien agrupaciones de dichas entidades. ${ }^{3}$

Estamos conscientes sin embargo de que tal constelación de regiones es resultado de la articulación de relaciones sociales, que han determinado a su vez las relaciones inter e intrarregionales, y de que sería necesaria una comprensión profunda de tales determinantes para poder explicar las diferencias efectivas observadas. No obstante, el objetivo concreto y limitado, por lo menos en esta etapa de nuestro estudio, consiste en conocer la evolución a largo plazo de la dinámica entre y dentro de las regiones.

Estamos de acuerdo con Lipietz en que mediante el análisis de la articulación de diversas etapas del modo de producción podría explicarse la evolución de la pobreza en las regiones de producción campesina y su interrelación con las regiones de tipo urbano-industrial del país. No obstante, un estudio a profundidad de tal naturaleza nos conduciria muy probablemente a regionalizaciones que poco tendrían que ver con la demarcación por la división político-administrativa del país y es de dudar que exista información estadística al nivel de desagregación adecuado.

Por tanto, en vista de su interés, así como por su facilidad operativa, continuaremos por ahora con el estudio de la disparidad interregional a partir de la medición de los resultados observables en las "regiones evidentes".

Con objeto de medir la desigual distribución de actividad económica entre estados y regiones, utilizamos el producto interno bruto (PIB) en cifras reales ${ }^{4}$ como indicador del nivel de dinamismo económico "real" estatal y/o regional. Al PIB en cifras constantes de 1970 (véase el cuadro 1), le calculamos una serie de coeficientes de concentración cuyas cualida-

${ }^{3}$ Alain Lipietz cuestiona la pertinencia de las regiones que resultan del corte por el espacio político administrativo cuando se intenta comprender las relaciones interregionales: “ . . . son las relaciones sociales las que, en la medida en que tienen una dimensión espacial, 'polarizan' el espacio social. La 'región' aparece así como el producto de las relaciones interregionales y éstas como una dirnensión de las relaciones sociales. No hay 'región pobre' sino sólo regiones de pobres, y si hay regiones de pobres es que hay regiones de ricos, y relaciones sociales que polarizan riqueza y pobreza, y las disponen en el espacio en forma diferencial" (Lipietz, 1979: 32).

${ }^{4}$ Empleamos cifras del PIB estatal elaboradas por el INEGI con el método del Sistema de Cuentas Nacionales, tanto para 1970 como para 1980 (véase por ejem- 
des para medir la desigualdad en una distribución tienen variantes de interés. $^{5}$

De acuerdo con los coeficientes estimados presentados en el cuadro 2 , podría concluirse que entre 1970 y 1980 disminuyó ligeramente la disparidad en el nivel de actividad económica entre estados. Sin embargo, un seguimiento detallado de los componentes concretos de la reducción nos permite distinguir dos tendencias en dirección contraria durante el decenio 1970-1980: la menor de ellas hacia una ligera desconcentración de la actividad económica y, la preponderante, hacia una creciente y, podríamos decir, segunda etapa de concentración.

En el primer caso figuran tres entidades tradicionalmente rezagadas cuyo PIB se incrementó a ritmos sorprendentes en la década pasada: Chia-

plo, INEGI 1984a) o bien, Cuaderno de Información Oportuna Regional, tercer trimestre, 1984. El PBB que figura en las publicaciones mencionadas de la SPP se presenta en términos nominales. En vista de que lo que interesaba era conocer la tendencia en la concentración de la actividad económica real, y el PIB estatal nominal de 1970 y 1980 no permite apreciar adecuadamente el nivel de dicha actividad para cada estado, intentamos aproximarnos al PIB estatal en 1980 en valores constantes de 1970 de la manera siguiente: construimos un índice de precios por estado mediante un promedio aritmético ponderado de los índices de precios sectoriales a nivel nacional de $1970-1980$, en donde las ponderaciones eran las participaciones relativas del PIB de cada sector dentro del PIB total del estado respectivo. Al calcular el índice de precios estatal de esta forma, se considera el peso de cada sector en el producto estatal:

$$
\text { Índice de precios } e=\frac{\sum_{i=1}^{7}\left(\text { Índice } \operatorname{precios}_{80,70}^{i}\right)\left(\mathrm{PIB}_{1980}^{i, e}\right)}{\sum_{i=1}^{7} \mathrm{PIB}_{1980}^{i, e}}
$$

e: $1,2, \ldots 32$ estados

i: $1,2, \ldots 7$ sectores agregados: agropecuario, mineria, manufactura, construcción, electricidad, distribución y resto de servicios.

Una falla evidente de nuestra estimación de los índices de precios estatales resulta de que las ponderaciones están dadas por el PIB relativo en cifras nominales; es decir, la ponderación misma ya está incluida por el efecto de los precios sectoriales. De haber tenido la información adecuada, tales ponderaciones deberían resultar del PB relativo en términos reales. No obstante, dado que con las ponderaciones se consideró el peso de cada sector en la producción estatal, creemos haber obtenido una buena aproximación de la tendencia de precios de cada estado.

Una vez deflactado el PIB de cada estado con nuestros índices de precios, la importancia relativa de la producción por estado dentro del PIB nacional varió de manera insignificante, excepto en los casos de Chiapas y Tabasco, en que la elevada importancia del petróleo, cuyos precios crecieron por encima de los de cualquier otro sector en la década pasada, provocaron que, al aislar la tendencia de precios, su PIB se redujera de modo notorio (véase el cuadro 1).

${ }^{5}$ Véase: Cortés y Rubalcava (1982). Los indicadores presentados fueron seleccionados por sus cualidades para permitir la cuantificación de la desigualdad según diversos criterios citados por los autores. 


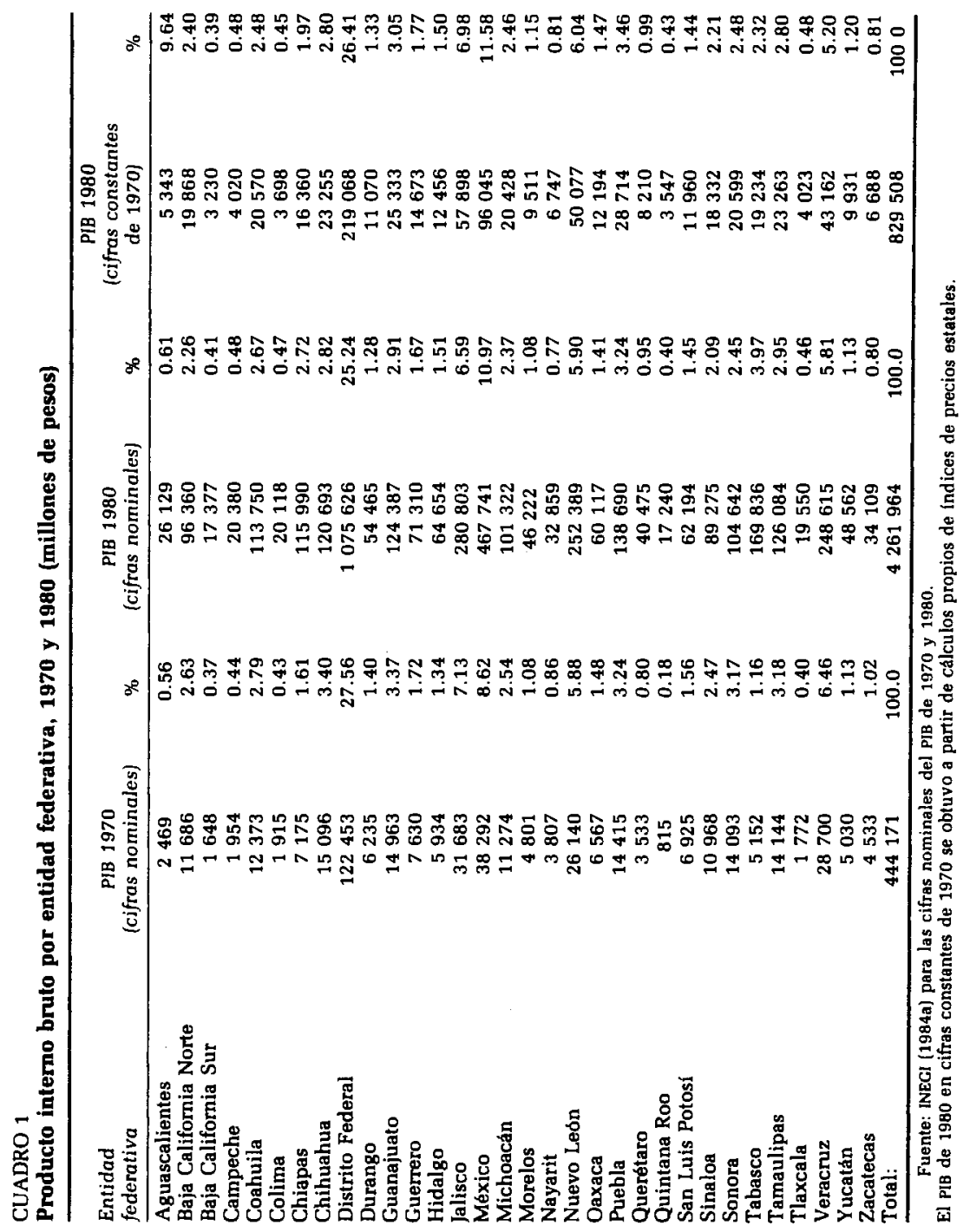


CUADRO 2

Coeficientes de concentración del producto interno bruto estatal ${ }^{*}$

\begin{tabular}{lll}
\hline & 1970 & $\mathbf{1 9 8 0}$ \\
\hline Varianza relativa & 0.077 & $\mathbf{0 . 0 7 5}$ \\
Desviación media relativa & 0.42 & $\mathbf{0 . 4 1}$ \\
Varianza logarítmica & 1.11 & $\mathbf{0 . 9 4}$ \\
Coeficiente de desigualdad de Theil & 0.185 & $\mathbf{0 . 1 8 2}$ \\
\hline
\end{tabular}

* Los indicadores presentados se estandarizaron para variar entre el intervalo cero-uno, donde el cero corresponde a la situación de igualdad total y la unidad a la concentración total La varianza logarítimica es la excepción, ya que no es posible dicha estandarización. Los coeficientes presentados en este cuadro se basan en la comparación del PIB de cada entidad federativa con aquel que se obtendría en una situación en que cada estado contribuyera en la misma magnitud al piB global del pais. La situación ideal de igualdad total que sirve como punto de comparación para medir la desigualdad, es aquella en que el PIB de cado estado fuese igual al PIB medio para el pais en su totalidad. Cuanto más se acerque el PIB de las distintas entidades hacia ese valor medio durante el periodo considerado, mas igualitaria se hace la situación.

pas, Tabasco y Quintana Roo, con 9, 14 y 16 por ciento repectivamente de crecimiento anual en términos reales, casos en los que el impulso dado por el Estado a la explotación petrolera y al turismo tuvo un papel muy importante. Desde luego queda abierta una interrogante en torno a si en algún futuro podrán crearse condiciones adecuadas para que el crecimiento petrolero o turístico se traduzca en mejores condiciones de vida para la población de las áreas mencionadas. Por ahora, la fuerte baja en el precio internacional del petróleo y la necesidad de impulsar las exportaciones no petroleras parece amenazar a entidades como Chiapas y Tabasco con una situación menos favorable que el decenio anterior en la capacitación de recursos federales para su expansión. En lo relativo a los coeficientes de concentración del PIB estatal, el crecimiento vigoroso del PIB de las tres entidades mencionadas disminuyó su diferencia respecto al PIB nacional, fenómeno que contribuyó a reducir el valor de los coeficientes de concentración entre 1970 y 1980.

En lo que se refiere al componente fundamental, lo calificariamos de creciente concentración, mediante un proceso de configuración de lo que podría denominarse una "región nodal", 6 caracterizado por:

a) El notable crecimiento de las entidades más cercanas al área urbana de la ciudad de México. Puebla, Tlaxcala, Querétaro, Morelos e Hidalgo elevaron su PIB 7, 9, 9, 7 y 8 por ciento anual, respectivamente (para

${ }^{6}$ Véase p.e. Arturo Ortega Blake (1984: 129): “La noción de región polarizada o región nodal. . . da más atención al impacto de las fuerzas producidas por el núcleo central sobre zonas satélites. . . Determina la interdependencia (relación, conectividad) y la jerarquía (dependencia) de las diferentes partes que componen una región." 
la economía en su totalidad, el PIB creció $6.5 \%$ anual en términos reales). Llegar a una delimitación de la región nodal a que nos referimos requeriría un trabajo muy complejo que no hemos abordado aún, pero el crecimiento de estas entidades sugiere la estructuración de un espacio económico crecientemente articulado en torno a un polo o nodo industrial: el valle de México. Un análisis estadístico posterior, respecto a la regionalización de Bassols, ${ }^{7}$ sustenta esta idea. El fuerte crecimiento de estas entidades acercó su PIB al PIB promedio del país, razón por la que disminuyó el coeficiente de concentración entre 1970 y 1980. Dado que no calificaríamos esta disminución como desconcentración, se convierte en una evidencia de la falla de medir la desconcentración regional con base en las entidades federativas como unidades de análisis, a pesar de lo cual decidimos conservarla en la presentación de los resultados porque permite comprender mejor los resultados con las entidades federativas agregadas en regiones más amplias.

b) Otro elemento que contribuyó a disminuir los coeficientes de concentración pero que representa una creciente concentración fue el crecimiento vigoroso del PIB del Estado de México: $10 \%$ de incremento anual en términos reales durante el decenio en estudio. El crecimiento industrial ha desbordado desde hace tiempo los límites del área urbana de la ciudad de México hacia el Estado de México y desde luego no se trata de ninguna desconcentración. De nuevo, este fenómeno acercó el PIB del Estado de México al PIB promedio nacional y disminuyó el coeficiente de concentración, lo que evidencia la falla de medir la concentración-desconcentración regional con base en entidades federativas.

En resumen, mediante el análisis de la concentración-desconcentración de la actividad económica con el PIB estatal, sólo el vigoroso crecimiento de Chiapas, Tabasco y Quintana Roo puede calificarse como con tendencia a la desconcentración, aunque estos estados tienen muy poco peso (relativo y absoluto) considerados en la producción total del país.

\section{Producto interno bruto per cápita por entidades federativas}

En el apartado anterior concluimos que la disminución de los coeficientes de concentración del PIB estatal correspondía fundamentalmente al crecimiento vigoroso del PIB de entidades que están paulatinamente articulándose en torno a un centro industrial: el del valle de México. Aunque este fenómeno se traducía en coeficientes de concentración decrecien-

${ }^{7}$ Este autor hace una interesante presentación de los factores naturales y sociales en la delimitación de las regiones de México, entre ellas las que estamos nencionando, que denomina: región centro-sur (Bassols, 1970). 
tes, representa más bien una mayor concentración por la estructuración de una "region nodal"; por tanto, la disminución de los coeficientes de concentración evidenciaba las deficiencias de considerar a los estados del país como regiones.

Sin cambiar aún de unidad de análisis, ahora tomaremos en cuenta (véase el cuadro 3) la concentración de actividad económica, así como la de población: nos encontramos de nuevo con coeficientes que indican una concentración que disminuye entre 1970 y 1980 y presentamos en seguida un análisis minucioso de los principales determinantes de dicha disminución para concluir, igual que en el apartado anterior, que no se trata sustancialmente de ninguna tendencia al equilibrio entre los estados del país, en lo que a actividad económica y población se refiere, Los principales elementos que explican la disminución de la distancia entre los PPC estatales y el PPC medio para el pais, durante 1970-1980, podrian resumirse como sigue:

\section{CUADRO 3}

\section{Coeficientes de concentración}

\section{del PIB per cápita estatal}

\begin{tabular}{lll}
\hline & \multicolumn{1}{c}{1970} & \multicolumn{1}{c}{1980} \\
\hline Variación relativa & 0.0074 & 0.0065 \\
Desviación media relativa & 0.16 & 0.15 \\
Varianza logarítmica & 0.16 & 0.13 \\
Coeficiente $\mathrm{R}_{n}^{m}$ & 0.0074 & 0.0065 \\
Coeficiente de Gini & 0.27 & 0.24 \\
\hline
\end{tabular}

a) Entidades tradicionalmente rezagadas que en la década 1970-1980 presentaron un elevado crecimiento de su PIB; éste sobrepasó el ritmo de crecimiento de su población, la cual aumentó a una tasa similar a la de la población de todo el país. Ejemplos: Tabasco, Chiapas y Puebla.

b) Entidades en las que existe un fuerte dinamismo económico pero que durante la década pasada concentraron población a un ritmo superior al que concentraron producto. Ejemplos: Distrito Federal, Estado de México y Nuevo León.

c) Entidades tradicionalmente rezagadas, cuya baja tasa de crecimiento de la población sugiere un fenómeno de emigración hacia fuera de la entidad. Ejemplos: Oaxaca y Zacatecas (véanse los cuadros 4 y 5 para los coeficientes de Gini, 1970 y 1980, así como el cuadro 6).

Dejando por el momento el caso a, podemos observar que las entidades fuertemente concentradoras de producción siguieron siéndolo en 1970-1980 y las tradicionalmente rezagadas (Oaxaca y Zacatecas, por ejem- 


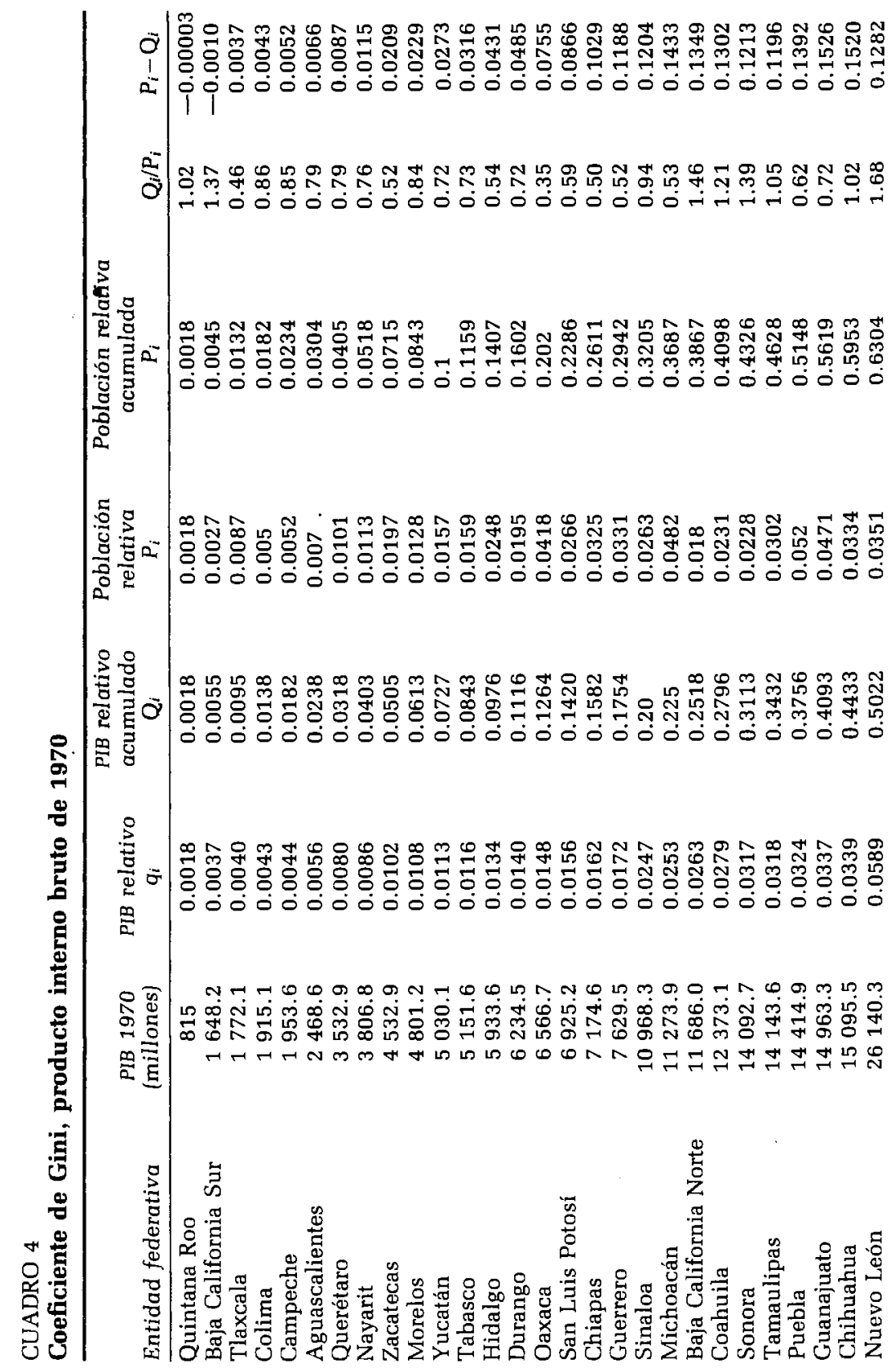



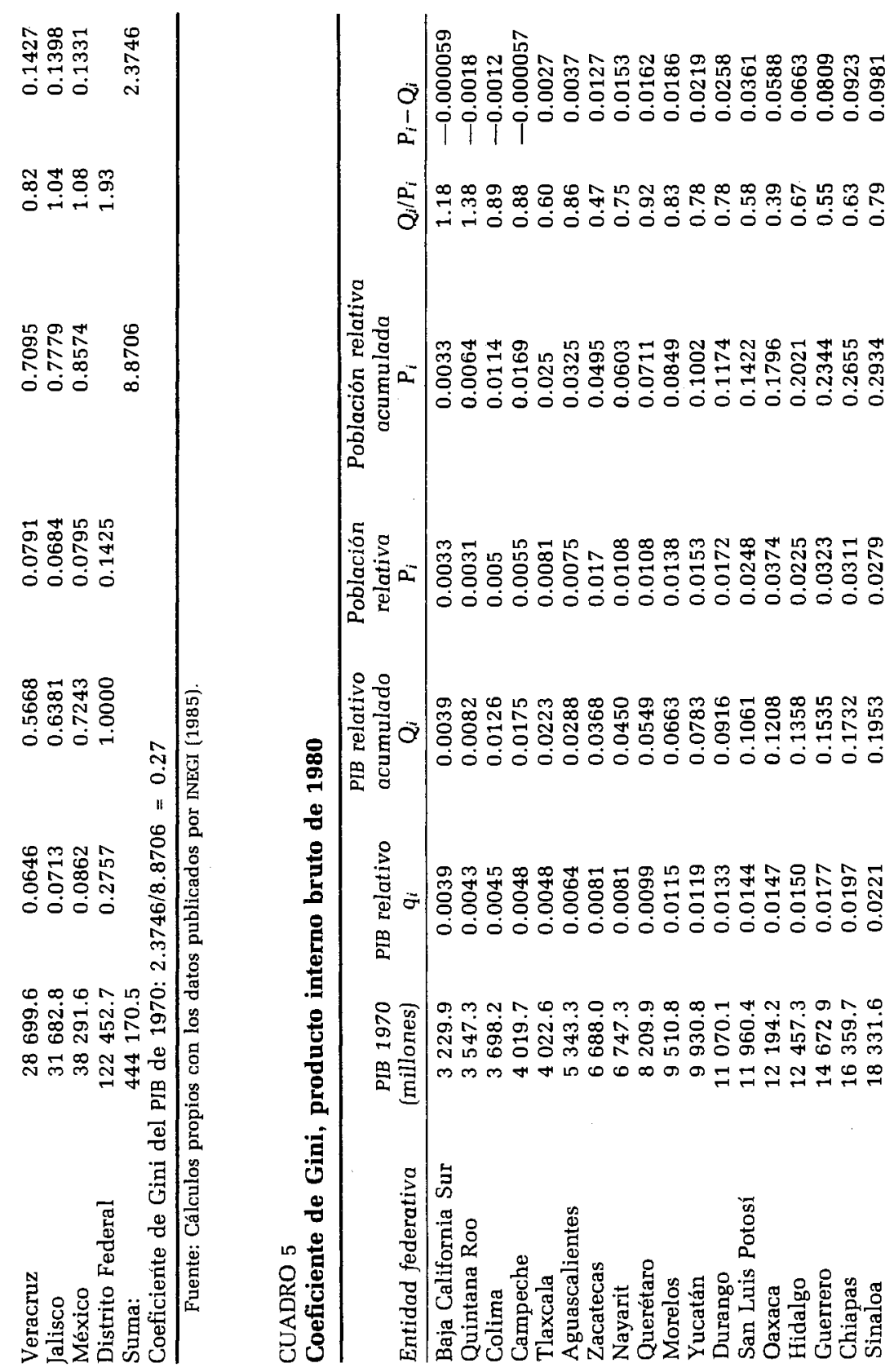


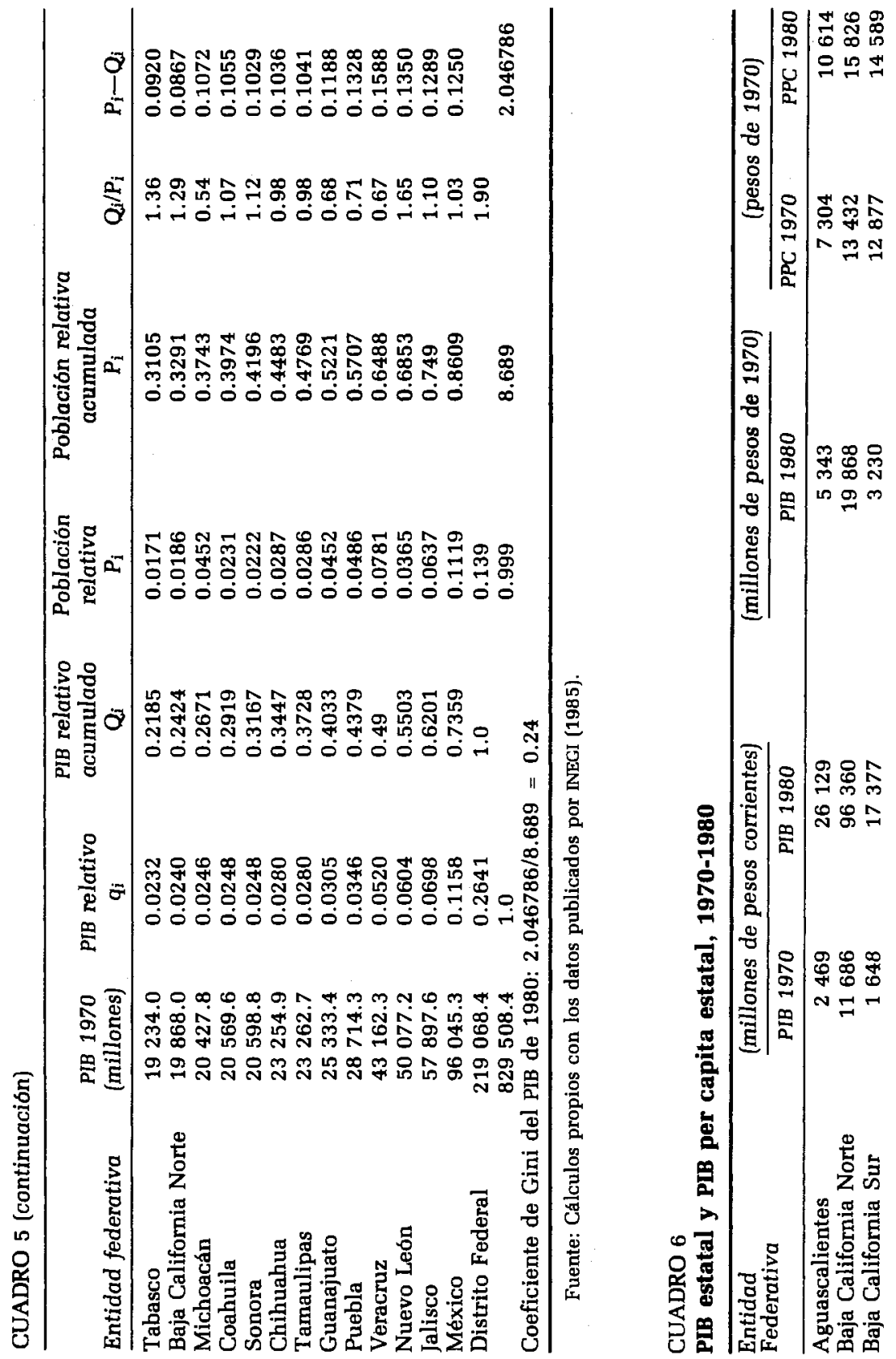




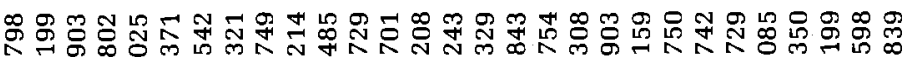

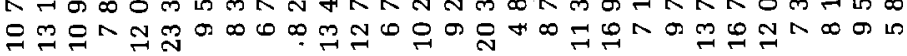

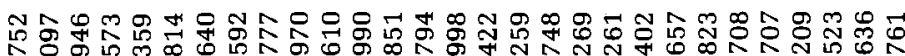

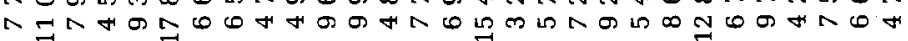

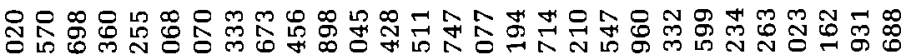

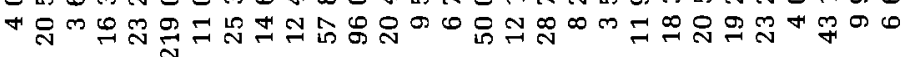

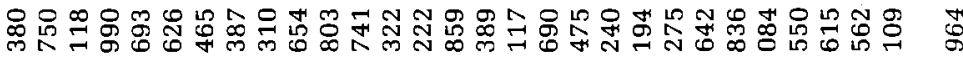

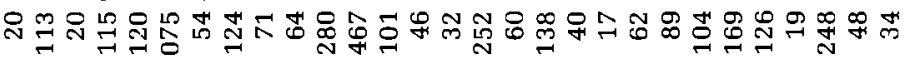

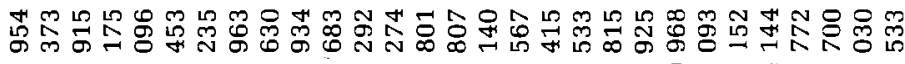

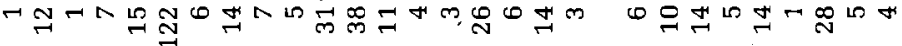


plo) también conservaron su rezago en la producción. Es decir, la disminución en la disparidad entre los PPC estatales se debe, en lo fundamental, a fenómenos demográficos: áreas rezagadas que disminuyen relativamente su población y áreas urbanas muy congestionadas por la inmigración.

Bajo una perspectiva neoclásica ${ }^{8}$ esta disminución en la desigualdad entre los niveles de PPC estatales permitiría sin duda concluir que entre 1970 y 1980 se ha iniciado un proceso de convergencia interregional:

En realidad, muchos de los aspectos de las consecuencias derivadas de esta migración [la interregional] apenas son motivo de divergencias entre quienes promueven otras teorías alternativas. La mayoría admitirá que la migración entre las áreas es un proceso de reajuste tendiente a reducir el desequilibro en los mercados locales (Richardson, 1976: 79).

Es decir, aceptando que existe una tendencia natural que lleva a los trabajadores a emigrar desde las zonas de salario bajo a las de salario alto y de las de elevado desempleo a las de escaso desempleo, con el tiempo se igualarían los niveles de salarios regionales, así como los índices de ocupación. Existe abundante evidencia empírica que refuta los modelos de migración neoclásicos ${ }^{9}$ así como los señalamientos unuy claros de las fallas lógicas de su base explicativa. ${ }^{10}$

Es muy probable que la disminución relativa de población en Oaxaca y Zacatecas se explique por un fenómeno de emigración que, hasta el momento no parece haber propiciado la acumulación de excedentes ni favorecido una expansión agrícola o industrial en las entidades que modifique su nivel de atraso relativo. ${ }^{11}$ Entonces, aun cuando el comportamiento del

${ }^{8}$ H. W. Richardson afirma: "La teoría neoclásica de equilibrio general establece que, en una situạción caracterizada por diferencias interregionales de salarios reales, la mano de obra emigra de las regiones con salarios bajos a las de salarios altos, hasta que éstos se equiparan. Tal conclusión se basa en diversos supuestos críticos: estructura estática comparativa; mano de obra homogénea; rendimientos a escala constantes; costos de emigración cero; mercados laborales perfectamente competitivos; traslado de los trabajadores como respuesta a las diferencias salariales y no por otra razón" (Richardson, 1976: 80).

${ }^{9}$ Stuart Holland (1976) cita variados estudios para las zonas del sur de Italia y del sur de los Estados Unidos.

${ }^{10}$ Alain Lipietz señala: "Si se parte de las hipótesis neoclásicas. . . los factores desigualmente repartidos tienden a desplazarse hasta la igualación de las productividades marginales de los factores, y por lo tanto de las tasas de ganancia y de salario. . . Como se trata de estática comparativa, el modelo no nos dice si esa uniformación termina por transformar en desiertos a las regiones demasiado poco capitalistas o por uniformar la distribución del capital. Observemos simplemente que este teorema neoclásico es perfectamente tautológico: si todo es móvil (entre regiones) y ello sin costo alguno, todo debe ser uniforme, y en realidad cabe preguntarse por qué se habla de economía regional" (Lipietz, 1979: 99).

${ }^{11}$ En sus modelos de migración aplicados al caso de Italia, Vera Lutz argu- 
PPC de dichas entidades haya contribuido a hacer disminuir los coeficientes de concentración, no reconocemos en esta tendencia ningún proceso de disminución de las desigualdades interestatales.

En contrapartida a las entidades federativas que expulsan población, ${ }^{12}$ las grandes ciudades se encuentran altamente congestionadas tanto por el crecimiento natural de su población como por la incontrolable inmigración a ellas; por ejemplo, el área urbana de la ciudad de México (Distrito Federal y Estado de México) y Monterrey. De esta manera resulta que en el área urbana del valle de México y en el área urbana de Nuevo León, la concentración de población ha rebasado a la ya de por sí acentuada concentración de actividad económica. El resultado lógico es que el PPC del Distrito Federal, del Estado de México y de Nuevo León disminuyó su crecimiento entre 1970 y 1980, contribuyendo a explicar de modo muy importante la reducción de los coeficientes de concentración del PPC.

Como ya mencionamos, es discutible la explicación neoclásica $\bigcap^{\natural}$ adjudica a los movimientos migratorios el papel de mecanismos de ajus: entre las regiones del país. El crecimiento económico se ha polarizado en las áreas urbano-industriales, en algunas regiones de agricultura comercial moderna y, recientemente, en zonas petroleras. Es evidente que una causa muy importante del congestionamiento urbano en las áreas de inmigración de trabajadores es el rezago rural en las regiones de expulsión de población, y es muy posible que de continuarse con el patrón de crecimiento sectorial fomentado y fortalecido en los últimos cuatro decenios, el desempleo o subempleo rural no hará más que seguirse trasladando a las grandes ciudades con el consecuente hacinamiento de trabajadores en zonas urbanas marginales, donde el suministro de infraestructura social no sólo no ha sido planificado sino que ni siquiera ha podido resolverse la correspondiente a zonas de trabajadores ya establecidos en las grandes ciudades. ${ }^{13}$

menta que la emigración del sector agrícola de una región atrasada genera un aumento en la relación tierra-trabajo y en la productividad agrícola; esto a su vez permite la generación de excedentes que pueden ser invertidos para mecanizar la agricultura o industrializar la región. De este modo, la migración estaría contribuyendo a disminuir las disparidades entre productividades marginales y entre ingresos interregionales. Este trabajo es citado por Stuart Holland para señalar la "mitología" del modelo en contraste con la realidad del sur de Italia.

${ }^{12}$ En un análisis reciente, el Consejo Nacional de Población señaló el constante desplazamiento de los habitantes del país: más de la mitad de las entidades federativas expulsan más población de la que reciben, en tanto que $2 / 3$ partes de quienes abandonan su lugar natal se dirigen hacia la ciudad de México. Monterrey o Guadalajara, en detrimento de la productividad agraria así como de las condiciones generales de vida urbana (citado en la Conferencia sobre Población y Ciudades Pequeñas y Medianas en América Latina y el Caribe, febrero de 1986).

${ }^{13}$ Son muchos los casos que ejemplifican el impacto de la inmigración a las 
En síntesis, en lo que se refiere a la evolución del PPC estatal, sólo la mejoría en las entidades con fuerte expansión petrolera en 1970-1980 (Chiapas y Tabasco) podría distinguirse como un fenómeno de cierta importancia en la desconcentración regional, debido a que disminuyó su situación de rezago económico relativo. La caída de precios del petróleo puede significar, sin embargo, que tal expansión haya sido sólo un auge coyuntural.

\section{Las disparidades regionales: regiones como conjuntos de entidades federativas}

Entre las diverses limitaciones del estudio de la problemática regional a partir de la división en entidades federativas, señalamos una que introduce un sesgo muy evidente en los resultados observables: la separación arbitraria entre el Distrito Federal y el Estado de México. Esta falla concreta se puede reducir al agregar las entidades en grupos regionales más amplios; sin embargo, la pregunta surgirá en torno a cómo agrupar los estados, con base en qué criterio se pueden incluir unas entidades y excluir otras de cada región. Hay dos criterios frecuentes para delimitar las fronteras de un espacio regional, el de homogeneidad y el de nodalidad:

Con respecto al criterio de homogeneidad, las áreas se agrupan para formar una región si éstas son consideradas homogéneas con relación a algún elemento básico, que puede ser económico (niveles de renta per cápita similares, una industria dominante común a todas las subáreas, una situación de desempleo relativamente uniforme) o social y político (delimitación con arreglo a la identidad regional, áreas tratadas conjuntamente por razones históricas, etc.). . . En tanto que los conceptos nodales de regiones acentúan la diferenciación espacial intrarregional. Admiten que la población y las actividades económicas no estarán dispersas uniformemente en una región, sino concentradas en focos específicos de actividad o en sus alrededores. . . El criterio para la inclusión de un área pequeña dentro de una región está condicionado a que esta área tenga vínculos de interdependencia más fuertes con centros mayores de la región que con otros centros fuera de ella (Richardson, 1976).

Ocurre, por tanto, que con las regiones homogéneas se centra el análisis en la diferenciación con otras regiones del espacio nacional, en tanto que con las nodales se enfatiza el grado de diferenciación intrarregional.

áreas urbanas, resultando una degradación de las condiciones de vida de los inmigrantes; se pueden citar las condiciones de vida de los trabajadores inmigrantes a Londres en el siglo pasado (Marx, 1972), y los estudios sobre la inmigración a las ciudades industriales de Italia procedentes del sur (Holland, 1976). 
En el presente trabajo de cuantificación de las disparidades interregionales seleccionamos dos clasificaciones elaboradas pór especialistas en el tema: la de Ángel Bassols (1970) y de Unikel, Ruiz y Garza (1976), a las que nos referiremos como "regionalización Bassols" y "regionalización URG"' (veánse los cuadros 7 y 8).

A través de un elemento económico -el nivel de PIB per cápita regional-y de un instrumento estadístico ${ }^{14}$ se llevó a cabo una cuantificación de la homogeneidad intrarregional (menor intravarianza) y de lo que podría constituir una aproximación de la nodalidad regional (mayor heterogeneidad intrarregional). Se encontró de esta forma que la clasificación URG correspondía principalmente a regiones homogéneas (la intravarianza explicaba el $30 \%$ en 1970 y el $45 \%$ en 1980 respecto a la varianza total del PPC), en tanto que la de Bassols parecería más acorde al criterio de nodalidad en sus regiones (la intravarianza explicó el $61 \%$ en 1970 y el $65 \%$ en 1980). La región que podría calificarse como de mayor nodalidad en la regionalización Bassols fue la centro-este, que comprende entidades crecientemente articuladas en torno al nodo industrial del área urbana de la ciudad de México: Puebla, Hidalgo, Querétaro, Tlaxcala y Morelos.

Pese a que en las dos clasificaciones se detectan elementos de homogeneidad y de nodalidad al mismo tiempo, se puede decir que en términos del nivel de PPC regional, las regiones de URG son fundamentalmente homogéneas en comparación con la agrupación de Bassols, en tanto que en esta última parece predominar el criterio de nodalidad. Consideramos por tanto que las dos regionalizaciones ofrecen aspectos interesantes para medir la tendencia en las disparidades regionales, por lo que analizaremos los resultados haciendo uso de ambas.

\section{Concentración de actividad económica por regiones: PIB regional}

En la primera parte de este trabajo observamos que con los coeficientes de concentración del PIB estatal disminuía aparentemente el nivel de concentración de la actividad económica entre estados. Asimisno, señalamos que el elemento más importante en la susodicha disminución, que era el crecimiento acelerado del Estado de México, representaba realmente no una disminución sino un aumento de la concentración de actividad en el área urbana de la ciudad de México, fenómeno que evidenciaba la falla

${ }^{14}$ La varianza relativa del PPC como indicador de la disparidad total en actividad económica y población se desagregó en sus dos componentes: la intravarianza que mide la disparidad intra-región y la intervarianza que mide la disparidad interregiones. 


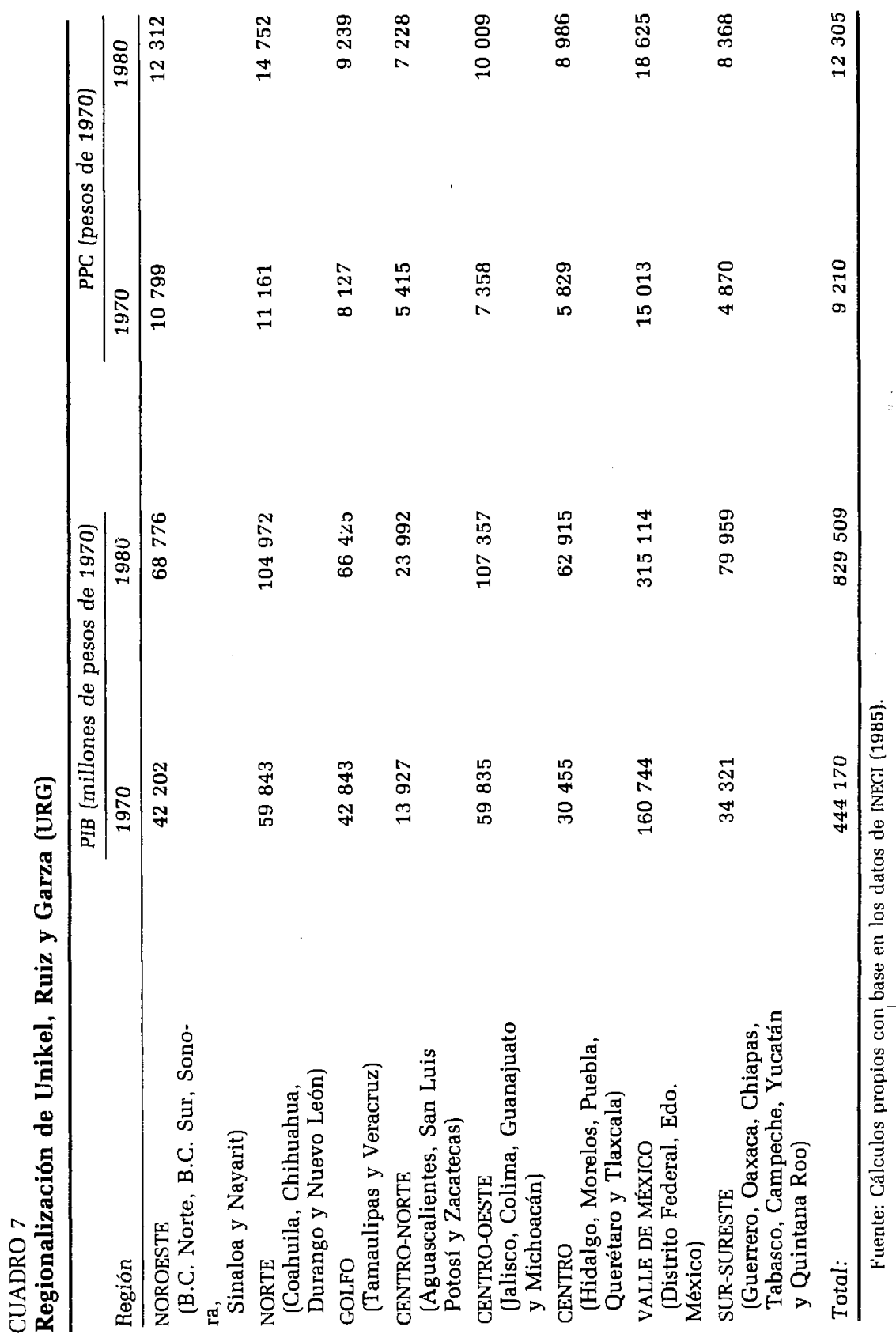


DESIGUALDADES INTERREGIONALES

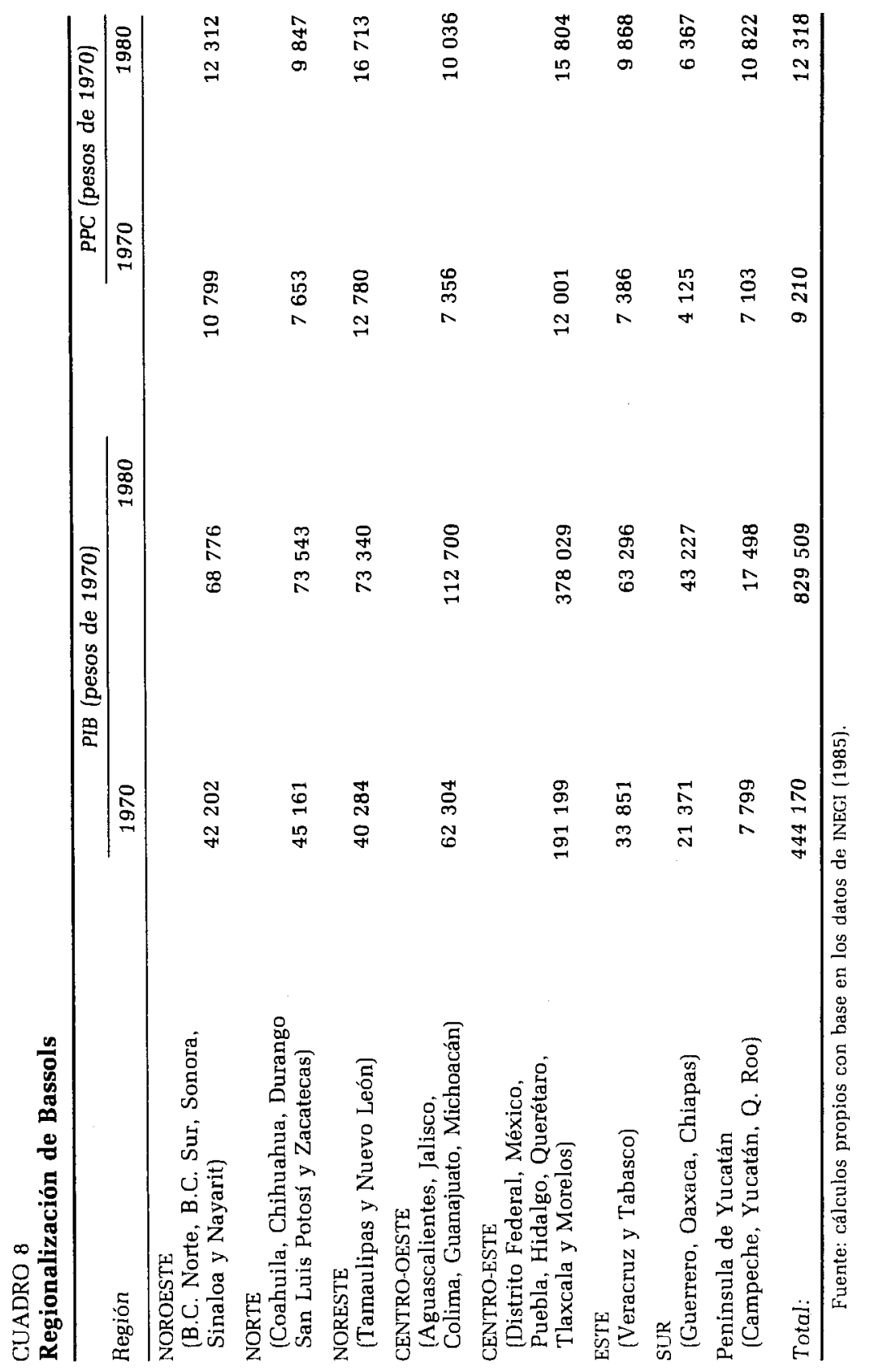


de considerar algunas entidades federativas como unidades regionales.

Disiparemos ahora la falla mencionada con la agregación de estados en unidades regionales más amplias. Concretamente, el Distrito Federal y el Estado de México quedarán en una sola región, con lo que el crecimiento acelerado de este último dejará de medirse erróneamente como el elemento de desconcentración.

Veremos nuevamente los resultados obtenidos con el PIB estatal para facilitar su comparación con los del nivel regional (véase el cuadro 9).

Se observa en el cuadro 9 que los coeficientes de concentración de la actividad económica medida con el PIB estatal reflejan una tendencia a la reducción de la concentración. Pero, como señalamos antes, tal disminución no es más que un error que resulta al considerar al Estado de México como unidad espacial separada del Distrito Federal. A pesar de que se detectaron elementos de lo que se podría denominar efectiva desconcentración a nivel estatal, como fueron el crecimiento acentuado de Tabasco, Chiapas y Quintana Roo, tienen una escasa incidencia en el valor de los coeficientes totales dado su poco peso relativo en el PIB nacional.

En el nivel regional, sin embargo, encontramos - a diferencia de lo detectado por Hernández Laos- ${ }^{15}$ que la actividad económica tendió a concentrarse más entre 1970 y 1980. Con las dos regionalizaciones empleadas aumentaron todos los coeficientes de concentración, excepto la varianza logarítmica con la de Bassols.

Con la regionalización URG resulta evidente la creciente desigualdad entre regiones. El Distrito Federal y el Estado de México, que constituyen la región valle de México, aumentaron su PIB relativo de $36 \%$ en 1970 a $38 \%$ en 1980. El PIB global de dicha región en 1970 fue 11.5 veces mayor que el de la región más atrasada (la centro-norte); para 1980 ya era 13 veces mayor. Por el lado de la reducción en la disparidad entre regiones juega un papel importante el crecimiento acelerado en la región sur-sureste, principalmente por la expansión petrolera en Chiapas, Tabasco y Campeche, y de los servicios turísticos en Quintana Roo. Sin embargo, el efecto neto observable en los coeficientes de concentración con la clasificación URG es de una mayor concentración regional, en vista de que la mayor concentración en el valle de México, por la importancia de esta región (38\% del PIB en 1980), opaca el elemento de desconcentración debido a la expansión en el sur-sureste.

Con la regionalización Bassols, el componente más relevante de la creciente concentración también está dado por el dinamismo sostenido del valle de México, sólo que ahora está comprendido dentro de una región más amplia, la región centro-este, que agrupa al Distrito Federal, Es-

${ }^{15}$ Mediante el análisis del PIB estatal y regional, así como del PIB percápita regional -empleando la regionalización URG - el autor detecta una incipiente convergencia interregional en la década 1970-1980. (Hernández Laos, 1984). 


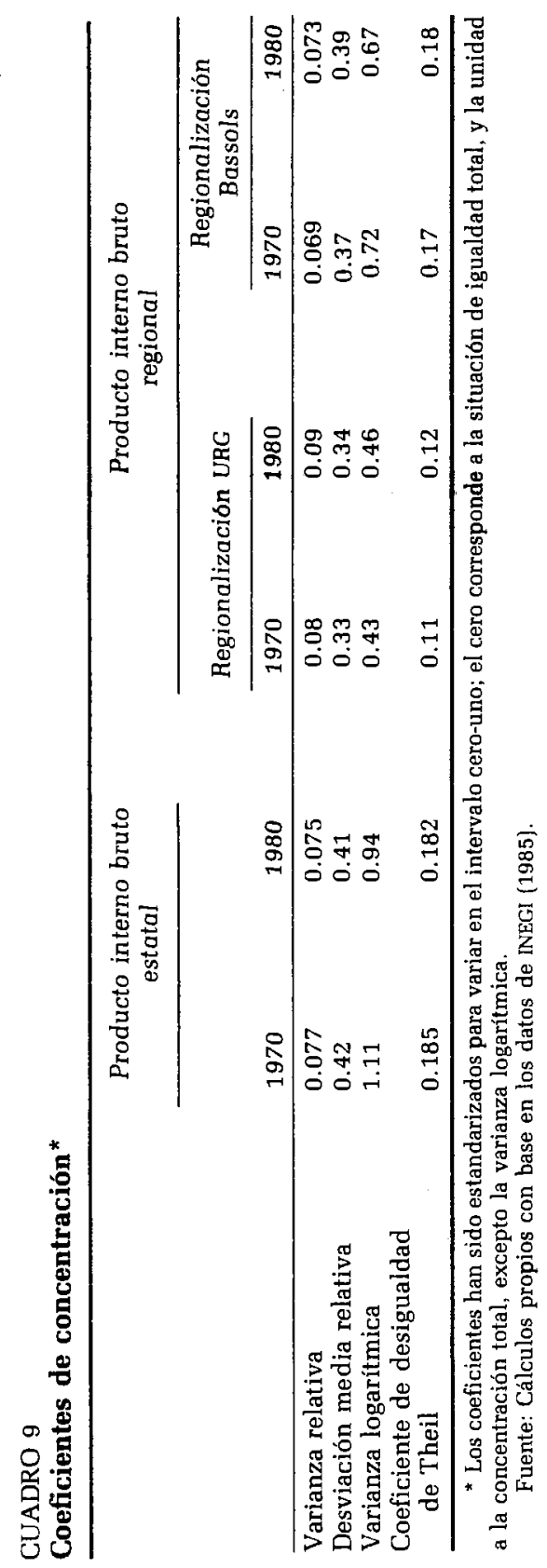


tado de México, Puebla, Hidalgo, Querétaro, Tlaxcala y Morelos. Esta región concentraba $43 \%$ del PIB en 1970 y para 1980 alcanzó $46 \%$ lo que se refleja en los valores crecientes de los coeficientes de concentración regional.

Los elementos de una efectiva, aunque incipiente, reducción en la desigualdad entre regiones son más notorios con esta regionalización que con la de URG. La amplia región sur-sureste de URG queda dividida en dos conjuntos con la clasificación Bassols: la península de Yucatán por un lado y la sur (Guerrero, Oaxaca y Chiapas) por otro, agrupando además a Tabasco en una región conjuntamente con Veracruz.

Por el menor nivel de agregación del sur-sureste, el crecimiento de Campeche y de Quintana Roo resulta más notorio; por lo tanto el crecimiento de estas regiones tradicionalmente rezagadas logra reflejarse haciendo disminuir la varianza logarítmica entre 1970 y 1980. Lo mismo sucede con la región sur; el acentuado crecimiento del PIB de Chiapas logra reducir la brecha entre la región sur, tradicionalmente la más rezagada de todas, y la más dinámica de todas que es la del centro.

En síntesis se puede afirmar que, tanto con la regionalización de URG como con la de Bassols, se observa una tendencia creciente en la disparidad entre regiones a través del comportamiento del PIB regional. El efecto de creciente concentración en el área metropolitana del valle de México y las entidades próximas contrarresta el efecto de reducción de concentración por la expansión en estados como Tabasco, Chiapas y Quintana Roo, resultando finalmente una creciente concentración regional en términos netos.

\section{Concentración de actividad económica y de población por regiones: PPC regional}

Como en el caso del PPC estatal, con la regionalización de URG y la de Bassols se observan coeficientes de una concentración regional decreciente entre 1970 y 1980 . Los determinantes de esta disminución, así como nuestra interpretación sobre si corresponden efectivamente o no a una desconcentración regional, son los siguientes:

a) Una región relativamente rezagada creció aceleradamente durante el decenio; la centro (Hidalgo, Morelos, Puebla, Tlaxcala y Querétaro). Desde el punto de vista de considerar esta zona como de rezago económico, que empieza a expandirse rápidamente, el fenómeno podría calificarse de disminución de las disparidades entre regiones. Sin embargo, mencionamos más arriba que este hecho parece sugerir un proceso de articulación de una zona nodal estructurada en torno al centro industrial del valle de México y, bajo esta perspectiva, el crecimiento acelerado de la región centro puede interpretarse más como de una nueva etapa de la 
concentración en torno al área metropolitana del valle de México que como una tendencia al equilibrio regional.

b) Otra región tradicionalmente rezagada que a pesar de una creciente población mostró un crecimiento notable en actividad económica fue la sur-sureste (Guerrero, Oaxaca, Chiapas, Tabasco, Campeche, Yucatán y Quintana Roo), aunque, como lo señalamos antes, el dinamismo de los estados que integran la región fue bastante dispar, en vista de que la expansión de Chiapas, Tabasco y Quintana Roo no se dio en las otras entidades del grupo. Este fenómeno, como ya afirmamos, podría calificarse como de una efectiva disminución de la disparidad entre regiones en virtud de que corresponde a la expansión productiva de zonas relativamente atrasadas económicamente.

c) Por último, el determinante más importante en la disminución de los coeficientes de concentración del PPC regional, lo constituye la desaceleración del crecimiento económico del valle de México. Esta región (Distrito Federal-Estado de México) tuvo uno de los crecimientos económicos más notables entre las distintas regiones del país; sin embargo, el crecimiento de su población fue el más elevado de todas: $4.7 \%$ de incremento anual promedio en su población en comparación con $3.4 \%$ de promedio nacional durante 1970-1980. Por tanto, el crecimiento del PPC de esta región, la más concentradora de actividad económica y de población, disminuyó y explica la reducción de los coeficientes de concentración del PPC regional.

En el apartado b señalamos que nos parece que aceptar los movimientos migratorios como mecanismos de ajuste de los desequilibrios regionales significa ignorar los determinantes de la polarización de rezago económico en las regiones que expulsan constantemente población hacia las áreas urbano-industriales del país, y tal conceptualización de la migración como factor de ajuste parecería corresponder más que nada a un deseo de encontrar una tendencia al equilibrio entre regiones que a nuestra realidad económico-espacial.

$\mathrm{Al}$ rechazar entonces el congestionamiento de población en el área urbana del valle de México como un factor equilibrador interregionalmente, podríamos concluir que la disminución de los coeficientes de concentración del PPC regional (veáse el cuadro 10) corresponde fundamentalmente a una creciente concentración de actividad económica y de población, excepción hecha del fenómeno de expansión en el sur-sureste.

Con la regionalización Bassols se observa asimismo una disminución de los coeficientes de concentración del PPC regional; sin embargo, tal como en el caso anterior, de los determinantes de la disminución sólo podemos considerar como factores de una leve tendencia a la reducción de las disparidades interregionales la expansión del sur y la de la península de Yucatán.

Quisiéramos concluir que, en lo que al PPC regional se refiere, e in- 


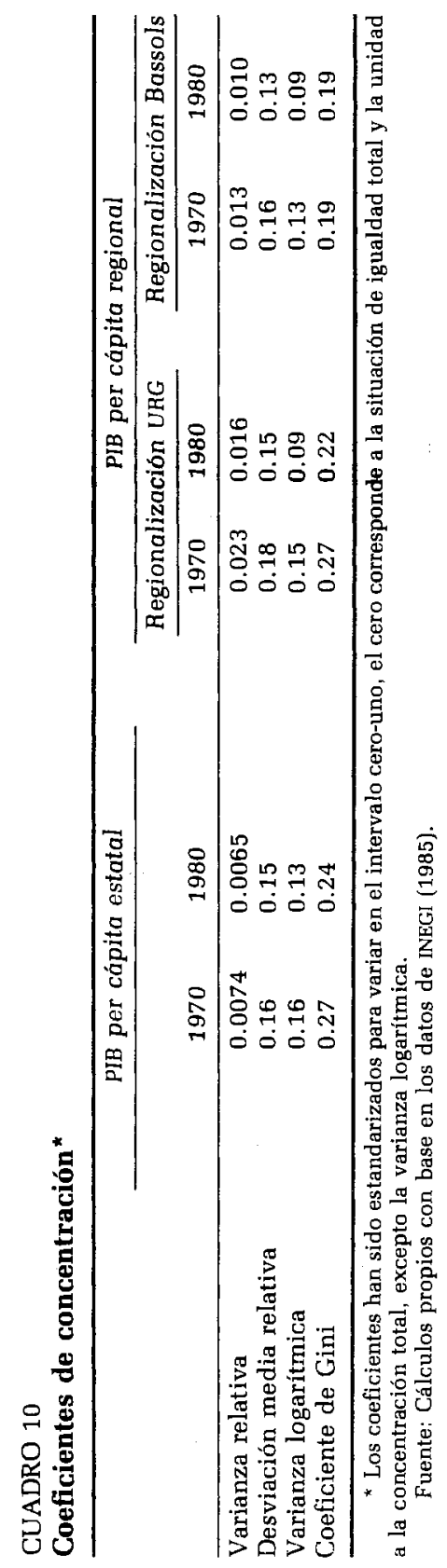


dependientemente de qué regionalización utilicemos de las dos analizadas, sólo la expansión petrolera en el sur-sureste, así como la de los servicios turísticos, nos dan elementos que reflejan una ligera disminución de la desigualdad entre regiones, pero persiste el elemento básico que mantiene una disparidad creciente: la expansión económica y de población en el valle de México y los estados de su entorno.

No existen investigaciones concluyentes para determinar si la concentración económica y poblacional observada ha sido o no propicia para el crecimiento económico del país. Dada la importancia del tema, sin embargo, es altamente deseable el estímulo de investigaciones que profundicen en el conocimiento de la distribución espacial de la población y de las actividades económicas en México y de su articulación al desarrollo económico nacional.

\section{Bibliografia}

Bassols Batalla, Ángel (1970), Geografía económica de México, Ed. Trillas, México. Cortés, Fernando y Rosa María Rubalcava (1982), Técnicas estadísticas para el estudio de Ia desigualdad social, El Colegio de México, México.

Hernández Laos, Enrique (1984), "La desigualdad regional en México", en varios, La desigualdad social en México, Siglo XXI, México.

Holland, Stuart (1976), Capital versus Regions, MacMillan, Londres.

INEGI (1984b), X Censo Nacional de Población, SPP, México.

INEGI (1985), Cuentas Nacionales por entidad federativa, SPP, México.

Cuentas Nacionales por entidad federativa, SPP, México.

Lemoine, John (1973), Causación acumulativa y crecimiento interregional en México, FCE, México.

Lipietz, Alain (1979), El capital y su espacio, Siglo XxI, México.

Marx, Karl (1972), EI capital, vol. 1, FCE, México.

Ortega Blake, Arturo (1984), La opción al cambio, Edicol, México.

Richardson, H.W. (1976), Teoría del crecimiento regional, Ed. Pirámide, Madrid.

Unikel, Luis, Crescencio Ruiz y Gustavo Garza (1976), EI desarrollo urbano de México, El Colegio de México, México.

Wilkie, James (1967), The Mexican Revolution: Federal Expenditure and Social Change since 1910, University of California Press. 
